Revue Sciences/Lettres

Sciences / Lettres

1 | 2013

Transferts culturels

\title{
Les inscriptions funéraires dans l'Italie préromaine
}

Marie-Laurence Haack

\section{(2) OpenEdition \\ 1 Journals}

Édition électronique

URL : http://journals.openedition.org/rsl/239

DOI : $10.4000 /$ rsl.239

ISSN : 2271-6246

Éditeur

Éditions Rue d'Ulm

Référence électronique

Marie-Laurence Haack, «Les inscriptions funéraires dans l'Italie préromaine », Revue Sciences/Lettres [En ligne], 1 | 2013, mis en ligne le 01 mai 2012, consulté le 02 mai 2019. URL : http:// journals.openedition.org/rsl/239; DOI : 10.4000/rsl.239

Ce document a été généré automatiquement le 2 mai 2019.

(c) Revue Sciences/Lettres 


\title{
Les inscriptions funéraires dans l'Italie préromaine
}

\author{
Marie-Laurence Haack
}

1 Sous les coups répétés de la New Archaeology, puis du courant post-processualiste, l'archéologie a depuis longtemps renoncé à fixer les contours d'une culture, et les archéologues sont devenus si réticents à utiliser les notions matérielles de culture et d'identité qu'ils s'intéressent désormais aux identités collectives et sociales plutôt qu'aux identités ethniques : si la culture matérielle demeure au centre de leurs préoccupations, elle est désormais appréhendée par le biais d'analyses sur les figures mouvantes des identités. Dans une Italie préromaine souvent décrite métaphoriquement comme une mosaïque d'identités, ce changement de perspective a des implications décisives : il a en effet permis de faire émerger des études sur les relations souvent méconnues entre les différentes parties non romaines de cette mosaïque et, par conséquent, de se débarrasser d'un point de vue romanocentriste. L'un des moyens d'observer les transferts à l'œuvre est de partir du domaine épigraphique, où, par force, noms propres, origines géographiques et relations interpersonnelles sont gravés dans une sorte d'instantané. On examinera ici les inscriptions du domaine funéraire parce que dans l'Italie préromaine, la plupart des inscriptions proviennent des nécropoles et que, loin de reproduire uniquement l'état-civil du défunt, lorsqu'elles sont inscrites sur du mobilier, elles témoignent aussi de ses relations extra-familiales. Nous étudierons ici une des communautés d'habitants de l'Italie préromaine les mieux connues en raison de l'énorme quantité d'inscriptions funéraires qu'elle a laissées, celle de Spina, un emporion situé dans la plaine du Pô, près de la bouche la plus au Sud du delta, et nous verrons si, à Spina, la présence d'étrangers a suscité des transferts culturels.

2 Si elles sont très nombreuses, les inscriptions de Spina sont pour la plupart très concises; mais les quelques mots - souvent deux tout au plus - qu'elles contiennent fournissent nombre d'informations sur les déplacements de personnes.

3 Un moyen de discerner l'installation d'étrangers consiste à se reporter à l'alphabet utilisé. Plusieurs défunts ou dédicants d'offrandes à des défunts sont en effet nommés en alphabet grec: Xanthippos dans la tombe 709 de Valle Trebba ${ }^{1}$, Tuchandros dans la tombe 
601 de Valle Trebba $^{2}$, Formionos dans la tombe 655B de Valle Pega, et Kritôn dans la tombe 630 de Valle Trebba ${ }^{3}$. Des divinités destinataires de dédicaces sont elles aussi nommées en grec et avec l'alphabet grec: Dionysos sur une assiette de la tombe 409 de Valle Trebba ${ }^{4}$, Apollon sur deux askoi, l'un de la tombe 650, l'autre de la tombe 779 de Valle Trebba ${ }^{5}$, et Hermès sur une autre assiette de la tombe 715 de Valle Trebba ${ }^{6}$. Quand la graphie est de type étrusque, l'onomastique peut être utilisée pour distinguer des écarts par rapport à des usages locaux. La tombe 623 de Valle Trebba abrite ainsi un petit plat marqué du nom de Platunalu ${ }^{7}$, appartenant probablement à un descendant d'immigré grec à Spina, puisqu'on reconnaît aisément dans ce nom une construction à partir du nom grec Platôn et du suffixe local-alus.

On aurait cependant tort de croire que les populations allogènes sont uniquement hellénophones : les Grecs sont alors les principaux partenaires commerciaux des Spinètes et la puissance dominante en Méditerranée, mais l'onomastique révèle aussi des transferts de populations italiques. De l'Ombrie voisine semblent venir Sekstalu ${ }^{9}$ et Pletine de la tombe 1173 de Valle Trebba $^{10}$. Enfin, il existe un certain nombre d'inscriptions où des gentilices vénètes, tels que Ostios, sont étrusquisés ${ }^{11}$.

D'un fait épigraphique et linguistique comme l'existence de noms d'origine étrangère, peut-on tirer des considérations sur l'idéologie de la communauté spinète ? En somme, une présence allogène implique-t-elle nécessairement des transferts de culture?

6 Les chercheurs ont longtemps mis la présence d'étrangers à Spina sur le compte d'une tolérance spinète, qui peut paraître étonnante étant donné que peu de cités d'Italie préromaine semblent avoir accueilli autant d'étrangers. Aussi un lien a-t-il été fait entre cette ouverture et l'absence de mention de charges administratives: pour nombre d'historiens, Spina ne peut pas avoir été une cité organisée, mais un port ou l'annexe d'une cité puissante, où résidaient des couches nouvelles ouvertes aux idées et aux hommes venus de l'étranger ${ }^{12}$.

7 On observera d'abord que la présence de populations d'origine étrangère est limitée en nombre. Les Grecs ou les Spinètes d'origine grecque repérables par l'épigraphie représentent une quinzaine de personnes. Quant aux populations d'origine italique, on n'en relève pas plus d'une dizaine de traces. En outre, on constate que la plupart de ces inscriptions, même celles de Spinètes aux noms tirés de noms de peuples voisins, sont tardives : elles datent pour la plupart d'entre 475 et 350 avant J.-C. Enfin, on note que le cas de Platunalu, Spinète dont un ancêtre du nom de Platôn a dû, pour pouvoir transformer son nom individuel en gentilice à consonance locale, obtenir la citoyenneté spinète, est isolé. La plupart des Grecs enterrés ou incinérés à Spina, contrairement aux habitudes locales, portent un seul nom, et sont nommés en grec sur des objets grecs. Ainsi, le nom de Xanthippos est gravé sur deux oinochoai attiques. Quant à Hermès, il lui est destiné une dédicace divine inscrite sur une assiette attique à vernis noir de la fin du $\mathrm{v}$ ${ }^{e}$ siècle avant J.-C. On ajoutera qu'il a été fait usage de rites grecs dans les tombes où des inscriptions grecques ont été découvertes et que tous les noms grecs retrouvés en contexte funéraire, à l'exception d'un ${ }^{13}$, appartiennent à des hommes, alors que le corpus épigraphique spinète accueille beaucoup de femmes. L'ouverture aux Grecs était donc très limitée, et les unions mixtes semblent avoir été rares, d'après l'épigraphie. On peut penser que les Grecs admis dans la nécropole étaient des étrangers avec lesquels des Spinètes étaient liés par des relations d'hospitalité et de commerce. 
8 Dans le domaine linguistique, quelques cas de transferts sont indéniables. On pense évidemment au nom individuel vénète Usti, rendu par le prénom ou par le gentilice Ostios dans les inscriptions de Spina. La langue grecque, en revanche, semble avoir rencontré peu de succès. L'usage de l'alphabet grec est réservé aux Grecs à quelques exceptions près seulement : le gentilice Herineś est écrit deux fois sur un askos en céramique achrome de la tombe 1026 de Valle Trebba, daté de la fin du IV siècle avant J.-C., une fois selon les normes étrusques et une autre fois avec un sigma à quatre traits et un $\mathrm{h}$ a scaletta, soit selon des formes influencées par les habitudes grecques ${ }^{14}$, comme si le lapicide voulait signifier une double culture graphique, voire linguistique, dont on entrevoit des traces ailleurs. Ainsi, une assiette à vernis noir de la tombe 39 de Valle Trebba, datée de la fin du $\mathrm{IV}^{\mathrm{e}}$ siècle ou du début du $\mathrm{III}^{\mathrm{e}}$ siècle avant J.-C., sert peut-être de support à un même nom de famille, rédigé deux fois, l'une en alphabet étrusque $(P(l) a t i)$, l'autre en alphabet grec $(\beta \lambda \alpha)^{15}$, et dans la tombe 1173 de Valle Trebba, la formule de possession en étrusque de Petine ${ }^{16}$ débute par la lettre grecque $\pi$, comme si le scribe s'était trompé et s'était soudain rappelé qu'il devait écrire en étrusque et non en grec.

9 Surtout, les deux langues, voire les deux écritures, ne semblent pas avoir joui du même prestige. En effet, parmi les inscriptions spinètes, on relève 11 abécédaires de langue étrusque, soit presque $15 \%$ des abécédaires étrusques connus, mais aucun abécédaire de langue grecque ${ }^{17}$. La plupart de ces abécédaires étant incomplets, on ne peut penser que ces objets aient pu avoir un rôle pratique; bien au contraire, réduits à deux ou trois lettres, ils avaient un caractère allusif et devaient servir seulement à signaler la maîtrise de l'écriture ou l'attachement à une culture. Ce manque d'attrait pour la langue grecque est paradoxal, alors que les objets grecs et certains traits du mode de vie à la grecque rencontraient un énorme succès : les Spinètes aisés utilisaient de la vaisselle de banquet grec et buvaient du vin, des femmes spinètes filaient avec un epinetron.

10 On peut proposer quelques clefs pour expliquer cet écart quantitatif qui nous conduit à distinguer, dans une même communauté, transferts matériels et transferts linguistiques. Le banquet en soi n'est pas une pratique considérée comme grecque par beaucoup d'Étrusques. Ces derniers se font représenter sur les fresques de leurs tombes festoyant, allongés sur des lits. De plus, quelques indices nous amènent à penser que les Spinètes suivent en matière de banquet certains usages étrusques, ainsi, les épouses sont conviées aux banquets. Les Spinètes empruntent donc aux Grecs surtout un répertoire de vases de banquet, souvent imagés ${ }^{18}$, bref, des contenants plutôt qu'un contenu de rites, et les vases grecs attestent un niveau de luxe atteint plutôt que l'adhésion à certaines valeurs. Ainsi, deux askoi attiques à figure rouge du début du $\mathrm{IV}^{\mathrm{e}}$ siècle avant $\mathrm{J}$.-C., portant une dédicace à Apollon d'un certain Griphos, ont été retrouvés dans deux tombes différentes parmi du mobilier datant du siècle suivant. Il s'agit probablement d'objets thésaurisés parce que leur origine grecque leur donnait un supplément de valeur.

11 L'attachement à l'écriture étrusque n'est pas propre à Spina, mais il est commun à l'ensemble de l'Étrurie padane, qui rassemble près de la moitié des abécédaires étrusques. Si la proximité des Vénètes qui déposaient des tablettes à écrire dans leurs sanctuaires peut expliquer la valeur sacrée de l'écriture, elle ne suffit pas à rendre compte de la préférence des Spinètes pour les alphabets étrusques plutôt que grecs. Le choix de posséder des abécédaires en étrusque correspond peut-être au désir de conserver le lien avec le passé colonial étrusque de la cité. Selon toute vraisemblance, en effet, Spina est à l'origine une colonie composée d'Étrusques du Nord et du centre de l'Étrurie. 
12 L'épigraphie fournit donc des instruments d'analyse pour mesurer l'ouverture d'une société à des populations allogènes. Elle montre que la communauté de Spina, tout en entretenant des relations commerciales privilégiées avec les Grecs $^{19}$, ne s'est pas montrée disposée à réaliser autant de transferts culturels qu'on a souvent voulu le croire. Au contraire, les inscriptions funéraires révèlent une société hiérarchisée, où quelques Grecs sont admis dans les nécropoles, mais où les Spinètes empruntent à la culture grecque des images plutôt que des mots ou des lettres.

\section{NOTES}

1. Voir REE, 46, $\mathrm{n}^{\circ} 15$ (nous utilisons ici la numérotation de la Rivista di epigrafia etrusca (REE); de la revue des Studi Etruschi (SE) ; et de l'édition de H. Rix (éd.), Etruskische Texte, II, Tübingen, 1991 ( ET)).

2. Voir REE, 46, $\mathrm{n}^{\circ} 15$.

3. Voir REE, 46, $\mathrm{n}^{\circ} 18$.

4. Voir SE, 52, 1984, p. 175-177, $\mathrm{n}^{\circ} 3$.

5. Voir Giovanni Uggeri, Primo contributo all'onomastica spinetica, in Santoro, Ciro - Marangio Cesare (éd.), Studi storico-linguistici in onore di Francesco Ribezzo, Mesagne, 1978, p. 402-403, n 72 a et $b$.

6. Voir Salvatore Aurigemma, Il R. Museo di Spina, Ferrare, 1936, p. 10, tav. V.

7. Voir SE, 58, 1992 (1993), p. 275-276.

8. Voir le cas parallèle de Kraikalu à Marzabotto. Cf. ET-Fe 2.7.

9. Voir ET, Sp 2.71.

10. Voir ET, Sp 2.80.

11. Voir ET, Sp 2.66 ; Sp 2.67.

12. Voir, par exemple, Mario Torelli, Storia degli Etruschi, Rome-Bari, 1981, p. 190 ; Mauro Cristofani, Gli Etruschi del mare, Milan, 1983, p.101; Françoise-Hélène Massa-Pairault, "Contribution à la réflexion sur le catalogue et l'exposition », in Fernando Rebecchi (éd.), Spina e il delta padano. Riflessioni sul Catalogo e sulla Mostra ferrarese (Atti del convegno internazionale di studi "Spina : due civiltà a confronto », 21 gennaio 1994, Ferrare, 1994), Rome, "L'ERMA » di BRETSCHNEIDER, 1998, p. 147 et p. 153. La quasi-absence d'armes dans les nécropoles attesterait l'ouverture de Spina.

13. Voir ET, Sp 2.62 : Thaisi.

14. ET, Sp 2.57. Voir le commentaire de Adriano Maggiani, «Sulla paleografia delle iscrizioni di Spina ", in F. Rebecchi (éd.), Spina ed il delta padano, éd. citée, p. 227-234, spécialement p. 233. On hésite à mettre cette inscription en relation avec le mot her, rédigé en grec, sur une assiette attique à vernis noir de la tombe 199 de Valle Trebba.

15. Voir ET, Sp 2.32.

16. Voir $E T$, Sp 2.80 .

17. Nous nous appuyons sur le catalogue de Maristella Pandolfini et Aldo Luigi Prosdocimi (éd.), Alfabetari e insegnamento della scrittura in Etruria e nell'Italia antica, Biblioteca di « Studi Etruschi », 20, Florence, 1990, qu'il faut compléter à l'aide de la Rivista d'Epigrafia Etrusca.

18. Sur l'éventuelle priorité donnée aux vases sur les images qui sont apposées, voir François Lissarrague, "Spina : aspects iconographiques ", in F. Rebecchi (éd.), Spina e il delta padano, éd. 
citée, p. 67-75 et Fernando Gilotta, «Il mondo delle immagini », in Fede Berti, Maurizio Harari (éd.), Storia di Ferrara II : Spina tra archeologia e storia, Ferrare, 2004, p. 132-156.

19. Les liens commerciaux expliquent sans doute que Spina ait été autorisée à ériger un trésor dans le sanctuaire delphique.

\section{RÉSUMÉS}

L'article vise à montrer l'apport de l'épigraphie à l'analyse des transferts culturels. Il s'appuie sur le cas de Spina, communauté d'Étrurie padane riche en inscriptions funéraires, pour montrer l'importance de l'idéologie dans les dynamiques de transferts.

This article aims to show the importance of epigraphy in the analysis of cultural exchanges. It considers the case of Spina, a settlement of Padan Etruria in which many funerary inscriptions were found, in order to show the importance of ideology in the dynamics of such exchanges.

\section{INDEX}

Mots-clés : Étrurie, épigraphie, inscriptions, Spina

Keywords : etruria, epigraphy, inscriptions, Spina

\section{AUTEUR}

\section{MARIE-LAURENCE HAACK}

Professeur d'histoire ancienne, université de Picardie (IUF).

Parmi les publications :

Écritures, cultures, sociétés dans les nécropoles d'Italie ancienne, Bordeaux, 2009 (ouvrage collectif sous la direction de M.-L. Haack).

Prosopographie des haruspices romains, Pise-Rome, 2006.

Les Haruspices dans le monde romain, Bordeaux, 2003. 